\title{
Decrease in salivary lactoferrin output in chronically intoxicated alcohol-dependent patients
}

\author{
Napoleon Waszkiewicz ${ }^{1}$, Beata Zalewska-Szajda², Anna Zalewska ${ }^{3}$, \\ Magdalena Waszkiewicz ${ }^{4}$, Sławomir Dariusz Szajda ${ }^{5}$, Bernadeta Repka ${ }^{6}$, \\ Agata Szulc ${ }^{1}$, Alina Kępka ${ }^{7}$, Alina Minarowska ${ }^{8}$, Sylwia Chojnowska ${ }^{9}$, \\ Beata Konarzewska ${ }^{1}$, Jerzy Robert Ładny ${ }^{5}$, Urszula Kowzan ${ }^{1}$, Krzysztof Zwierz ${ }^{6}$ \\ ${ }^{1}$ Department of Psychiatry, Medical University of Bialystok, Poland \\ ${ }^{2}$ Department of Imaging Diagnostics, Medical University of Bialystok, Children's Hospital, Poland \\ ${ }^{3}$ Department of Pedodontics, Medical University of Bialystok, Poland \\ ${ }^{4}$ Neurology Unit, Regional Hospital in Bialystok, Poland \\ ${ }^{5}$ Department of Emergency Medicine and Disasters, Medical University of Bialystok, Poland \\ ${ }^{6}$ Medical College of the Universal Education Society, Lomza, Poland \\ ${ }^{7}$ Department of Biochemistry and Experimental Medicine, \\ the Children's Memorial Health Institute, Warsaw, Poland \\ ${ }^{8}$ Department of Anatomy, Medical University of Bialystok, Poland \\ ${ }^{9}$ Medical Institute, College of Computer Science and Business Administration, Lomza, Poland
}

\begin{abstract}
Salivary lactoferrin is a glycoprotein involved in the elimination of pathogens and the prevention of massive overgrowth of microorganisms that affect oral and general health. A high concentration of lactoferrin in saliva is often considered to be a marker of damage to the salivary glands, gingivitis, or leakage through inflamed or damaged oral mucosa, infiltrated particularly by neutrophils. We conducted a study to determine the effect of chronic alcohol intoxication on salivary lactoferrin concentration and output. The study included 30 volunteers consisting of ten non-smoking male patients after chronic alcohol intoxication (group A), and 20 control nonsmoking male social drinkers (group C) with no history of alcohol abuse. Resting whole saliva was collected 24 to 48 hours after a chronic alcohol intoxication period. Lactoferrin was assessed by enzyme-linked immunosorbent assay. For all participants, the DMFT index (decayed, missing, or filled teeth), gingival index (GI) and papilla bleeding index (PBI) were assessed. The differences between groups were evaluated using the Mann-Whitney U test. We noticed significantly decreased salivary flow (SF) in alcohol dependent patients after chronic alcohol intoxication (A), compared to the control group (C). Although there was no significant difference in salivary lactoferrin concentration between the alcohol dependent group A and the control group C, we found significantly decreased lactoferrin output in group A compared to group C. We found a significant correlation between the amount of daily alcohol use and a decrease in lactoferrin output. There was a significant increase in GI and a tendency of PBI to increase in group A compared to group C. We demonstrated that chronic alcohol intoxication decreases SF and lactoferrin output. The decreased lactoferrin output in persons chronically intoxicated by alcohol may be the result of lactoferrin exhaustion during drinking (due to its alcohol-related lower
\end{abstract}


biosynthesis or higher catabolism) or to decreased function of neutrophils affected by the ethanol. The poorer periodontal state in alcohol dependent persons compared to controls may be a result of lower salivary flow and decreased protection of the oral cavity by lactoferrin. (Folia Histochemica et Cytobiologica 2012, Vol. 50, No. 2, 248-254)

Key words: alcohol, ethanol, saliva, lactoferrin

\section{Introduction}

Almost $4 \%$ of global deaths, $4.6 \%$ of diseases and injuries, and $30 \%$ of health care costs, are attributed to alcohol abuse [1]. Alcohol drinking is the world's third largest risk factor for disease and disability, and in middle-income countries it is the greatest risk factor [2]. About $2 \%$ of the world-wide population is dependent on alcohol [3]. Ethanol is directly toxic to the mucosa of the oral cavity, mouth, throat, esophagus and stomach. Acute local mucosa damage is reversible and proportional to the concentration of alcohol. Ethanol immediately diffuses into saliva and oral tissues, reaching in saliva and oral tissues higher concentrations than in plasma [4-9]. Acetaldehyde, the main metabolite of ethanol, also damages oral tissues [10]. However, oral tissue damage may also be the result of a direct action of ethanol, reactive oxygen species (ROS) generated by ethanol and its metabolites, and fatty acid ethyl esters (FAEEs). Oral mucosa is the portal of entry for many pathogens. The saliva and system of innate and acquired immunity of the oral mucosa eliminate the majority of pathogens and prevent massive overgrowth of microorganisms. Salivary proteins participate not only in protection of the oral cavity, but also in protection of the upper gastrointestinal and respiratory tracts [9-12].

Lactoferrin is a multifunctional glycoprotein of the transferrin family that participates in the innate immune response. It is secreted by the intercalated duct cells of salivary glands [7,13]. Lactoferrin is found on the surface of mucous membranes, in the granules of polymorphonuclear leukocytes, neutrophils, and in biological fluids such as milk, saliva, tears and seminal fluid [13]. An increase in lactoferrin extracellular concentration is often regarded as an indicator of neutrophil activation $[14,15]$. A high lactoferrin concentration in saliva is often considered a marker of damage to the salivary glands, gingivitis, or leakage through inflamed or damaged oral mucosa, infiltrated by neutrophils [16]. As iron cations are an essential nutrient for certain types of bacteria, two binding sites for iron cations in lactoferrin provide its bacteriostatic effect by limiting availability of iron for bacteria such as Streptococcus mutans [17, 18]. Through direct interaction with the bacterial porins, lactoferrin exerts its bactericidal effect $[13,15]$. Lactoferrin interacts with endotoxin (LPS, lipopolysaccharide) of the Gram-negative bacterial membrane, thus releasing the LPS from the membrane [13]. The consequence of subsequent LPS binding by the lactoferrin is reduced synthesis of LPS-mediated tumor necrosis factor (TNF- $\alpha$ ) that protects against septicemia [19]. Lactoferrin also participates in the inhibition of bacterial adhesion to tooth surfaces, aggregation of bacteria, and the activation of phagocytic cells. Lactoferrin has also antifungal, antiviral (prevents infection by binding to the host cell), antiparasitic (reduces the integrity of the parasite membrane and its interaction with host tissues, binds parasite nutrients i.e. iron cations), as well as immunomodulatory action (increases production of IL-1, IL-2, and increases activity of monocytes/macrophages, granulocytes and NK cells) and anti-inflammatory properties [7, 17, 20]. Lactoferrin is partially digested in the gastrointestinal tract by pepsin and trypsin to lactoferricin, a cationic peptide that exhibits antimicrobial activity in the lower gastrointestinal tract [21]. This would support the hypothesis that salivary proteins besides the oral cavity are also involved in protection downstream along the gastrointestinal tract.

It has been reported that there is a reduction in the stimulated release of lactoferrin after acute in vitro effect of ethanol on neutrophils [22], a tendency towards an increase in serum lactoferrin concentration in alcohol dependent patients [14], and no effect of acute alcohol intoxication on salivary lactoferrin concentration [7]. The objective of the present study was to determine for the first time the effect of chronic alcohol consumption on the concentration and output of salivary lactoferrin.

\section{Material and methods}

Subjects. The study included 30 volunteers comprising ten male alcohol-dependent non-smoking patients (group 'A', standing for alcohol) admitted to the Detoxification Unit of the Psychiatric Hospital in Choroszcz, Poland, after chronic alcohol intoxication (mean age: 49 years; range: $39-57$ ) (100-200 g/day of alcohol), and 20 control male social drinkers (group 'C', standing for control) with no history of alcohol abuse or smoking (mean age: 42 years; range: $30-53$ ). Alcohol dependent individuals in group A met the criteria for alcohol dependence according to ICD-10 (the average 
time of dependence was $12 \pm 8$ years). The length of alcohol intoxication ranged from three to 30 days (mean $~ 8$ ). Material from alcohol dependent persons admitted to the Detoxification Unit was collected on the second day of the abstinence period.

Ethical issues. This study was approved by the Bioethical Committee of the Medical University of Bialystok, Poland (R-I-003/289/2005). Informed written consent was obtained from all the participants after explanation of the nature, purpose, and potential risks of the study.

Data and sample collection. A check-up of the oral cavity was done by one qualified dentist in artificial light, using a dental mirror and a probe. Following the World Health Organization criteria, the level of dental caries in subjects was determined using the DMFT index (decayed, missing or filled teeth; [7]). Gingival status was assessed using the gingival index (GI; Löe and Silness [23]) and papilla bleeding index (PBI; [24]). The DMFT, GI, and PBI indices of our subjects were: in alcohol dependent participants (20.6 \pm $\pm 3.5,1.58 \pm 1.04,0.93 \pm 0.65$, respectively), and in controls $(18.8 \pm 5.70,0.30 \pm 0.47,0.35 \pm 0.48$, respectively $)$. The subjects were instructed to refrain from food and beverages, except water, for two hours before the collection of saliva. All salivary samples (3 $\mathrm{ml}$ of resting whole saliva) were collected to plastic tubes placed on ice by the spitting method, under standardized conditions [25, 26], between 8.00 am and $9.00 \mathrm{am}$, to minimize the influence of circadian rhythms. The saliva samples were centrifuged at 3,000 $\times \mathrm{g}$ for 20 minutes at $4^{\circ} \mathrm{C}$, to remove cells and debris. The resulting supernatants were divided into $200 \mu$ l portions, frozen and kept at $-80^{\circ} \mathrm{C}$, until analyzed. Salivary flow (SF) was calculated by dividing the volume of saliva by the time of its collection. In all samples, the lactoferrin concentration was assayed in duplicate, and the means of the duplicate results were used as final values.

Lactoferrin assay. An enzyme-linked immunosorbent assay (ELISA) method was used to determine the salivary lactoferrin concentration, as previously described [7], In brief, MaxiSorp ${ }^{\mathrm{TM}}$ microplates (Nunc Brand cat. no 449824, Roskilde, Denmark) were coated with rabbit anti human lactoferrin (primary) antibody 1:500 (Sigma, L3262, St Louis, MO, USA) and blocked with $2 \%$ bovine serum albumin (BSA; Sigma, A7030). Lactoferrin from human milk (Sigma, L0520) was applied as a standard. Saliva samples [diluted 1:10 and 1:20 in phosphate-buffered saline (PBS) containing $0.75 \%$ BSA and $0.1 \%$ Tween 20], $50 \mu \mathrm{l} /$ well], were applied onto blocked plates. Then secondary antibodies 1:15,000 (rabbit anti-human lactoferrin antibody; polyclonal antibody biotinylated, ICN, 673581, Costa Mesa, CA, USA) were applied. The microplates with secondary antibodies were incubated with horseradish peroxidase (HRP) conju-

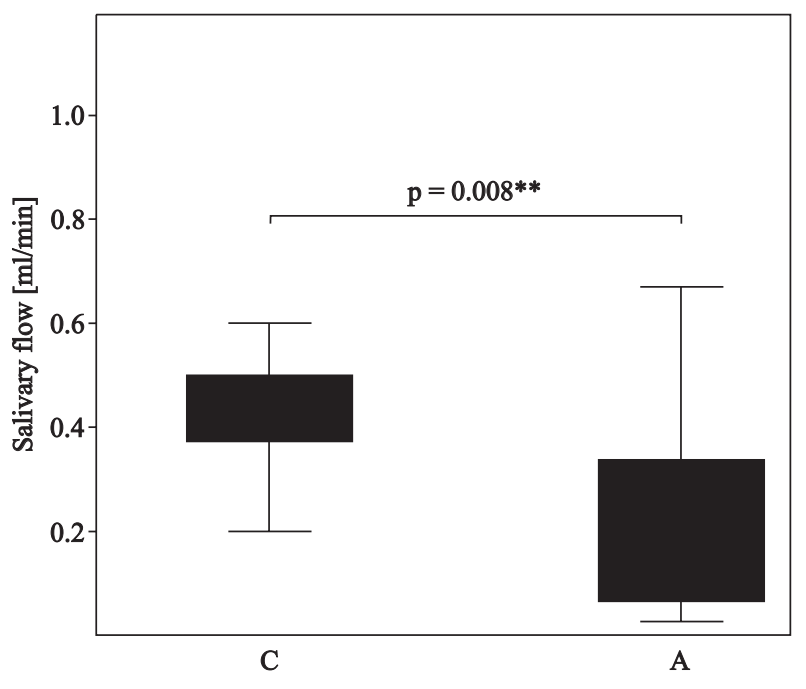

Figure 1. Salivary flow (SF) in control social drinkers (C) and alcohol dependent persons (A)

gated with streptavidin (Dako, P0397, Glostrup, Denmark) 1:2,000 in PBS. Streptavidin has a high affinity for biotin. HRP bound to streptavidin is ready for color development after addition of the substrate, o-phenylenediamine (OPD). HRP reaction was stopped by adding $0.5 \mathrm{M} \mathrm{H}_{2} \mathrm{SO}_{4}$, $50 \mu \mathrm{l} /$ well. Color development was measured at $450 \mathrm{~nm}$ (Elx800 ${ }^{\mathrm{TM}}$, Bio-Tek Instruments, Inc., Winooski, VT, USA) and was proportional to the quantity of lactoferrin in the sample. As negative controls, two wells were incubated for 30 minutes with PBS instead of sample/standard, two wells were processed without primary antibody, and two without secondary antibody. Results were analyzed by the KC Junior program. Bio-Tek software applications include KC4, IBM, and KC Junior for MS-DOS (all manual and automated readers).

Statistical analysis. Statistical analysis was performed using Statistica version 9.0 (Statsoft, Krakow, Poland). Results are expressed as means $\pm \mathrm{SD}$. The differences between groups were evaluated using the Mann-Whitney U test. Statistical significance was assumed at $\mathrm{p}<0.05$.

\section{Results}

Salivary flow (SF) was significantly lower in chronically intoxicated alcohol dependent patients $(0.23 \pm$ $\pm 0.24)$ than in the control social drinking group $(0.43 \pm 0.10 \mathrm{ml} / \mathrm{min})\left(\mathrm{p}=0.008^{* *}\right)$ (Figure 1). No significant differences were found between the concentration of lactoferrin in the saliva of alcohol dependent patients $(4.53 \pm 2.56 \mathrm{mg} / \mathrm{l})$ and the control social drinkers $(4.48 \pm 2.92 \mathrm{mg} / \mathrm{l})(\mathrm{p}=0.929)$. Salivary lactoferrin output was significantly decreased in 


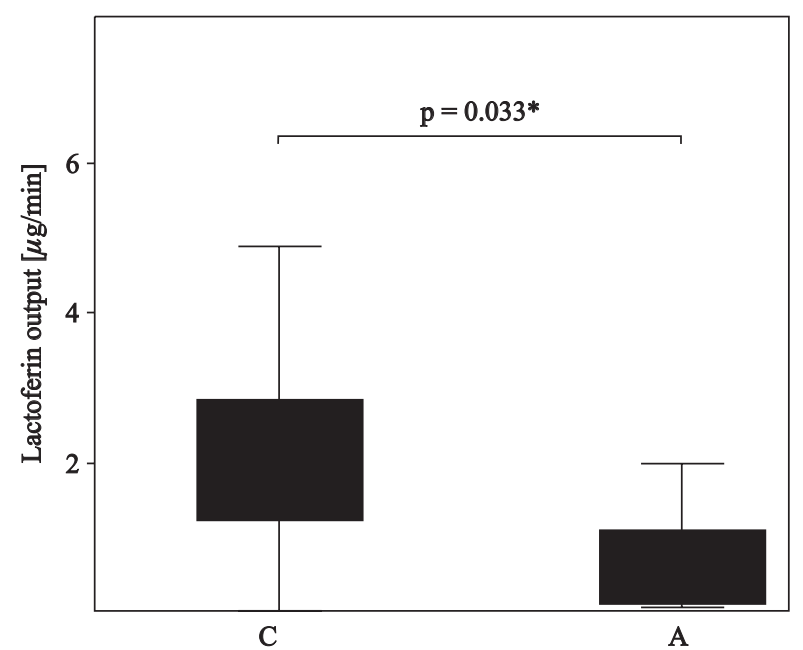

Figure 2. Lactoferrin output in control social drinkers (C) and alcohol dependent persons (A)

Table 1. Effect of chronic ethanol intoxication (A) on the DMFT (decayed, missing or filled teeth) index, GI (gingival index) and PBI (papilla bleeding index), compared to the control social drinkers $(\mathrm{C})$

\begin{tabular}{|l|l|c|c|}
\hline Variable & \multicolumn{1}{|c|}{$\mathbf{C}$} & $\mathbf{A}$ & $\mathbf{p}$ \\
\hline DMFT & $18.8 \pm 5.7$ & $20.6 \pm 3.5$ & 0.355 \\
\hline GI & $0.30 \pm 0.47$ & $1.58 \pm 1.04$ & $0.029^{*}$ \\
\hline PBI & $0.35 \pm 0.48$ & $0.93 \pm 0.65$ & 0.081 \\
\hline
\end{tabular}

Values are expressed as the mean $\pm \mathrm{SD} ; \mathrm{p}<0.05$ considered statistically significant: ${ }^{*} p<0.05,{ }^{* *} p<0.01,{ }^{* * *} p<0.001$; p: C to A group

alcohol dependent persons chronically intoxicated by ethanol $(0.74 \pm 0.74 \mu \mathrm{g} / \mathrm{min})$ compared to the control group $(1.93 \pm 1.35 \mu \mathrm{g} / \mathrm{min})\left(\mathrm{p}=0.033^{*}\right)$ (Figure 2$)$. Gingival index (GI) was significantly higher in alcohol dependent persons $(1.58 \pm 1.04)$ than in the control group $(0.30 \pm 0.47)\left(\mathrm{p}=0.029^{*}\right)$ (Table 1$)$. Papilla bleeding index (PBI) had a high tendency to increase in alcohol dependent persons $(0.93 \pm 0.65)$ compared to the control group $(0.35 \pm 0.48)(\mathrm{p}=$ $=0.081)$. There was a weak tendency of DMFT
Table 3. Spearman's rank correlation coefficient between salivary flow (SF) and the DMFT (decayed, missing or filled teeth) index, GI (gingival index) and PBI (papilla bleeding index), in alcohol dependent subjects

\begin{tabular}{|l|c|c|c|}
\hline Variable & DMFT & GI & PBI \\
\hline $\mathrm{SF}[\mathrm{ml} / \mathrm{min}]$ & $\mathrm{r}^{2}=-0.098$ & $\mathrm{r}^{2}=-0.423$ & $\mathrm{r}^{2}=-0.419$ \\
& $\mathrm{p}=0.630$ & $\mathrm{p}=0.038^{*}$ & $\mathrm{p}=0.041^{*}$ \\
\hline
\end{tabular}

$\mathrm{p}<0.05$ considered statistically significant: ${ }^{*} \mathrm{p}<0.05,{ }^{*} \mathrm{p}<0.01$, $* * * p<0.001$

index (decayed, missing or filled teeth) to increase in alcohol dependent persons $(20.6 \pm 3.5)$ than in the control group $(18.8 \pm 5.7)(\mathrm{p}=0.355)$. There was a significant correlation between the amount of daily alcohol consumption and the output of salivary lactoferrin $\left(r^{2}=0.819, p=0.045^{*}\right)$ (Table 2). There were no significant correlations between the duration of alcohol consumption and salivary lactoferrin output/ /concentration. We also did not find any correlations between amount/duration of alcohol intoxication, SF, lactoferrin concentration/output, and the DMFT, GI, and PBI indices, in group A. Significant inverse correlations were found between SF and PBI $\left(r^{2}=-0.419\right.$, $\mathrm{p}=0.041)$, as well as between SF and GI $\left(\mathrm{r}^{2}=-0.423\right.$, $\mathrm{p}=0.038)($ Table 3$)$.

\section{Discussion}

After chronic ethanol consumption, the following have been reported: fat accumulation, acinar cell swelling, salivary gland atrophy, reduced salivary flow rate, markedly decreased salivary protein and glycoprotein levels/outputs (e.g. immunoglobulin A) [6-8, $27,28]$. However, little is yet known as to the possible effects of chronic ethanol intoxication on the level of salivary lactoferrin. Previous studies concerning alcohol's effect on lactoferrin have reported a reduction in stimulated release of lactoferrin after acute action in vitro of ethanol on neutrophils [22], a tendency to increase in serum lactoferrin concentration in alcohol dependent patients [14], and no effect of

Table 2. Spearman's rank correlation coefficient between amount/duration of alcohol intoxication and lactoferrin concentration/output, in alcohol dependent subjects

\begin{tabular}{|l|c|c|}
\hline Variable & Amount of alcohol per day $[\mathrm{g}]$ & Duration of alcohol intoxication (days) \\
\hline Lactoferrin concentration $[\mathrm{mg} / \mathrm{l}]$ & $\mathrm{r}^{2}=0.698$ & $\mathrm{r}^{2}=0.206$ \\
& $\mathrm{p}=0.122$ & $\mathrm{p}=0.696$ \\
\hline Lactoferrin output $[\mu \mathrm{g} / \mathrm{min}]$ & $\mathrm{r}^{2}=0.819$ & $\mathrm{r}^{2}=0.205$ \\
& $\mathrm{p}=0.045^{*}$ & $\mathrm{p}=0.695$ \\
\hline
\end{tabular}

$\mathrm{p}<0.05$ considered statistically significant: ${ }^{*} \mathrm{p}<0.05,{ }^{*} \mathrm{p}<0.01,{ }^{* * *} \mathrm{p}<0.001$ 
acute alcohol intoxication on the salivary lactoferrin concentration [7].

Although we did not find a significant difference between the concentration of lactoferrin in the saliva of alcohol dependent persons and in that of control social drinkers, salivary lactoferrin output was significantly decreased in alcohol dependent persons compared to the social drinkers $(\mathrm{p}=0.033)$ (Figure 2). Since alcohol abuse has been found to decrease some glycoprotein biosynthesis, modification and transport, and to increase other glycoprotein secretion, elimination and catabolism [6], the decreased lactoferrin output may also be the result of subsequent lactoferrin exhaustion during a long drinking period. As we noted a high correlation between the amount of daily alcohol consumption and salivary lactoferrin output (Table 2), ethanol may exert a directly toxic effect on lactoferrin secretion. It should be mentioned that salivary lactoferrin is secreted not only by intercalated duct cells of salivary gland tissue [7, 13], but may also be released by activated neutrophils in damaged salivary glands, in gingivitis, or may be the result of blood leakage through inflamed or damaged oral mucosa $[16,29]$. It has been reported that six hours after acute ethanol (40\%) consumption, damage had been caused to the oral mucosa epithelium with degeneration of the epithelial cells and leukocyte infiltration; after 12 hours, irreversible cytolysis or swelling of the intermediate layer cells of the oral mucosa developed, whereas 24-48 hours after acute alcohol consumption, disseminated ulcerations developed in the mouth $[7,30,31]$. After induced oral tissue damage (e.g. by alcohol), one of the first lines of defense against pathogens is the tissue recruitment of neutrophils [32]. It has been shown that alcohol intoxication generally inhibits the recruitment and activity of neutrophils and macrophages as well as their hydrogen peroxide production $[33,34]$. This is consistent with the results of our study, as we noted significantly decreased salivary lactoferrin output in chronically intoxicated alcohol dependent persons.

Since lactoferrin is a marker of neutrophil activity, its reduced salivary output may suggest a deleterious effect of chronic alcohol intoxication on neutrophil activity, even if neutrophil infiltration occurred. Our study is not consistent with the one by Nilsson et al. [22] who observed no changes in the spontaneous secretion of lactoferrin by neutrophils acutely treated in vitro by ethanol. This could however be due to low levels of ethanol $(1 \%)$ or too short a duration of alcohol action (10 minutes) to change the cellular metabolism of lactoferrin (our participants drank 5-40\% alcohol for 3-30 days). The lack of changes in lactoferrin concentration has been previously de- scribed after acute alcohol intoxication in saliva [7], and after chronic alcohol intoxication in serum [14].

Our results of decreased lactoferrin output, compared to Thome et al. [14], suggest that the salivary glands and oral cavity may be much more sensitive to chronic alcohol intoxication than serum. There is nothing surprising in that, since ethanol diffuses rapidly into saliva during drinking, and immediately after drinking ethanol salivary concentration is temporarily much higher than in plasma [9], suggesting that ethanol easily penetrates to the salivary glands. After alcohol intake, the level of acetaldehyde in saliva strikingly exceeds the level in systemic blood. From saliva, acetaldehyde, ethanol, and other metabolites of alcohol and toxins such as reactive oxygen species (ROS) or fatty acid ethyl esters (FAEEs), easily reach all local tissues [5-9]. The lack of lactoferrin concentration decrease in our earlier acute alcohol intoxication study [7] might be the result of the lack of salivary flow data. After chronic alcohol intoxication, we also found no changes in lactoferrin concentration. But when compared to the SF data, we found decreased lactoferrin output. Salivary flow is the main factor affecting salivary composition, which varies in accordance with the type, intensity, and duration of the stimulus [17]. As an increase in SF generally is accompanied by an increase in the concentration of total protein, sodium, calcium, chloride, bicarbonate, and $\mathrm{pH}$ level in the saliva [17], the reduced SF level in our study might also reduce the output of lactoferrin.

In our study, SF was significantly decreased in persons chronically intoxicated by alcohol, compared to control social drinkers (Figure 1). Our findings agree with the alcohol-induced SF reduction observed in experimental studies [35]. Our results are also consistent with the study reported by Dutta et al. [36], in which chronic alcohol intoxication resulted in a reduction in the stimulated SF from parotid glands. The SF reduction in patients suffering from alcohol dependence may be due to morphological changes of the salivary glands, since in these persons enlarged or atrophied salivary glands associated with accumulation of fat in the secretory cells, reduction of the weight of the salivary glands, and inflammatory infiltration of the parenchymal space around salivary ducts, have all been reported [7, 27, 37-39]. In alcohol abusers, ethanol replaces water from hydrogen bonds of hydrophilic domains of proteins, which increases the fluidity of parenchymal cell membranes of salivary glands. Replacement of water by ethanol impairs the activation of cAMP in the membrane, $\mathrm{Ca}^{2+}$ mobilization, and finally impairs the secretory process [5-9]. The decrease in SF may also be the result of alcohol withdrawal syndrome stress. Alcohol 
withdrawal syndrome stress is characterized by a significant central and peripheral sympathetic hyperactivity, hypercortisolemia, reduced food intake and fluid diet (which reduces mastication that stimulates SF), and reduced number of muscarinic receptors as well as reduced secretion/action of paracrine gastrointestinal hormones and transmitters such as somatostatin, PP peptide, and cholecystokinin [5, 9, 40-42].

The association between heavy alcohol drinking and poor oral hygiene is widely known [43]. Dental and oral hygiene are often neglected by persons addicted to alcohol $[8,9,44,45]$. It has also been found that persons addicted to alcohol with poor dental status have a doubled concentration of acetaldehyde in their saliva compared to those addicted to alcohol but who take care of their oral hygiene [43]. In alcohol abusers, reduced salivary flow (SF) may lead to inflammatory states of the oral cavity, periodontal diseases, or infections [46]. Although we found only a weak tendency to increase in DMFT index (decayed, missing or filled teeth) in alcohol dependent persons than in the control social drinkers, we found a significantly higher gingival index (GI) and a strong tendency of papilla bleeding index (PBI) to be higher in alcohol dependent persons than in the control social drinkers (Table 1). This suggests a significantly poorer periodontal state in alcohol dependent persons than in social drinkers, which may be the result of decreased salivary flow in alcoholics as significant inverse correlations were found between SF and PBI and between SF and GI (Table 3).

We have here demonstrated decreased salivary flow and lactoferrin output after chronic alcohol intoxication. Lower lactoferrin output in persons after chronic alcohol intoxication may be the result of lactoferrin exhaustion (due to its alcohol-induced lower biosynthesis or higher catabolism), or to decreased function of neutrophils affected by the ethanol. A poorer periodontal state in alcohol dependent persons than in social drinkers may be a result of lower salivary flow and decreased lactoferrin protection of the oral cavity.

\section{References}

1. Waszkiewicz N, Konarzewska B, Waszkiewicz M et al. Biomarkers of alcohol abuse. Part I. Traditional biomarkers and their interpretation. Psychiatr Pol. 2010;44:127-136.

2. World Health Organization. Global Status Report on Alcohol. 2011 Geneva, Switzerland.

3. Waszkiewicz N, Szulc A. Diagnosis of alcohol abuse. Przegl Lek. 2009;66:529-534.

4. Waszkiewicz N, Zalewska A, Szulc A et al. The influence of alcohol on the oral cavity, salivary glands and saliva. Pol Merkur Lekarski. 2011;30:69-74.
5. Waszkiewicz N, Szajda SD, Kępka A, Szulc A, Zwierz K. Glycoconjugates in the detection of alcohol abuse. Biochem Soc Trans. 2011;39:365-369.

6. Waszkiewicz N, Szajda SD, Zalewska A et al. Alcohol abuse and glycoconjugate metabolism. Folia Histochem Cytobiol. 2012;50:1-11.

7. Waszkiewicz N, Szajda SD, Jankowska A et al. The effect of acute ethanol intoxication on salivary proteins of innate and adaptive immunity. Alcohol Clin Exp Res. 2008;32:652-656.

8. Waszkiewicz N, Zalewska A, Szajda SD et al. The effect of chronic alcohol intoxication and smoking on the output of salivary immunoglobulin A. Folia Histochem Cytobiol. 2012; (in press).

9. Waszkiewicz N, Zalewska A, Szajda SD et al. The effect of chronic alcohol intoxication and smoking on the activity of oral peroxidase. Folia Histochem Cytobiol. 2012; (in press).

10. Waszkiewicz N, Poplawska R, Konarzewska B et al. Biomarkers of alcohol abuse. Part II. New biomarkers and their interpretation. Psychiatr Pol. 2010;44:137-146.

11. Waszkiewicz N, Szulc A. Immunity defects in acute and chronic alcohol intoxication. Pol Merkur Lekarski. 2010;172:269-273.

12. Waszkiewicz N, Szajda SD, Konarzewska B, Szulc A, Kepka A, Zwierz K. Underappreciated role of binge drinking in the risk of lung cancer. Eur J Public Health. 2010;20:6.

13. Farnaud S, Evans RW. Lactoferrin - a multifunctional protein with antimicrobial properties. Mol Immunol. 2003;40: 395-405.

14. Thome J, Foley P, Gsell W et al. Increased concentrations of manganese superoxide dismutase in serum of alcohol-dependent patients. Alcohol Alcohol. 1997;32:65-69.

15. Klebanoff SJ, Waltersdorph AM. Prooxidant activity of transferrin and lactoferrin. J Exp Med. 1990;172:1293-1303.

16. Almstahl A, Wikstrom M, Groenink J. Lactoferrin, amylase and mucin MUC5B and their relation to the oral microflora in hyposalivation of different origins. Oral Microbiol Immunol. 2001;16:345-352.

17. de Almeida Pdel V, Gregio AM, Machado MA, de Lima AA, Azevedo LR. Saliva composition and functions: a comprehensive review. J Contemp Dent Pract. 2008;9:72-80.

18. Jankowska AK, Waszkiel D, Kobus A, Zwierz K. Ślina jako główny składnik ekosystemu jamy ustnej. Część II. Mechanizmy odpornościowe. Wiad Lek. 2007;60:253-257.

19. Brock J. Lactoferrin: a multifunctional immunoregulatory protein? Immunol Today. 1995;16:417-419.

20. Rudney JD. Does variability in salivary protein concentrations influence oral microbial ecology and oral health? Crit Rev Oral Biol Med. 1995;6:343-367.

21. Amerongen AV, Veerman EC. Saliva - the defender of the oral cavity. Oral Dis. 2002;8:12-22.

22. Nilsson E, Hallden G, Magnusson KE, Hed J, Palmblad J. In vitro effects of ethanol on polymorphonuclear leukocyte membrane receptor expression and mobility. Biochem Pharmacol. 1996;51:225-231.

23. Loe H, Silness J. Periodontal disease in pregnancy. I. Prevalence and severity. Acta Odontol Scand. 1963;21:533-551.

24. Saxer UP, Muhlemann HR. Motivation and education. SSO Schweiz Monatsschr Zahnheilkd. 1975;85:905-919.

25. Dawes C. Physiological factors affecting salivary flow rate, oral sugar clearance, and the sensation of dry mouth in man. J Dent Res. 1987;66:648-653.

26. Navazesh M, Christensen C, Brightman V. Clinical criteria for the diagnosis of salivary gland hypofunction. J Dent Res. 1992;71:1363-1369. 
27. Riedel F, Goessler U, Hormann K. Alcohol-related diseases of the mouth and throat. Best Pract Res Clin Gastroenterol. 2003; 17:543-555.

28. Slomiany BL, Piotrowski J, Slomiany A. Chronic alcohol ingestion enhances tumor necrosis factor-alpha expression and salivary gland apoptosis. Alcohol Clin Exp Res. 1997;21:1530-1533.

29. Tanida T, Okamoto T, Okamoto A et al. Decreased excretion of antimicrobial proteins and peptides in saliva of patients with oral candidiasis. J Oral Pathol Med. 2003;32: 586-594.

30. Muller P, Hepke B, Meldau U, Raabe G. Tissue damage in the rabbit oral mucosa by acute and chronic direct toxic action of different alcohol concentrations. Exp Pathol. 1983;24:171-181.

31. Knoll MR, Kolbel CB, Teyssen S, Singer MV. Action of pure ethanol and some alcoholic beverages on the gastric mucosa in healthy humans: a descriptive endoscopic study. Endoscopy. 1998;30:293-301.

32. Fitzgerald DJ, Radek KA, Chaar M, Faunce DE, DiPietro LA, Kovacs EJ. Effects of acute ethanol exposure on the early inflammatory response after excisional injury. Alcohol Clin Exp Res. 2007;31:317-323.

33. Nelson S, Kolls JK. Alcohol, host defense and society. Nat Rev Immunol. 2002;2:205?209.

34. Goral J, Karavitis J, Kovacs EJ. Exposure-dependent effects of ethanol on the innate immune system. Alcohol. 2008;42:237-247.

35. Maier H, Born IA, Veith S, Adler D, Seitz HK. The effect of chronic ethanol consumption on salivary gland morphology and function in the rat. Alcohol Clin Exp Res. 1986;10: 425-427.

36. Dutta SK, Orestes M, Vengulekur S, Kwo P. Ethanol and human saliva: effect of chronic alcoholism on flow rate, com- position, and epidermal growth factor. Am J Gastroenterol. 1992;87:350-354.

37. Ryder MI. The influence of smoking on host responses in periodontal infections. Periodontol 2000. 2007;43:267-277.

38. Scott J, Burns J, Flower EA. Histological analysis of parotid and submandibular glands in chronic alcohol abuse: a necropsy study. J Clin Pathol. 1988;41:837-840.

39. Smith PF, Ramirez JC, Romo RE, Esguep AS. Salivary glands in alcoholism: a histologic and sialographic study. Addict Biol. 1996;1:385-393.

40. De Witte P, Pinto E, Ansseau M, Verbanck P. Alcohol and withdrawal: from animal research to clinical issues. Neurosci Biobehav Rev. 2003;27:189-197.

41. Karolewska E, Konopka T, Pupek M, Chybicka A, Mendak M. Antibacterial potential of saliva in children with leukemia. Oral Surg Oral Med Oral Pathol Oral Radiol Endod. 2008;105:739-744.

42. Keedwell PA, Poon L, Papadopoulos AS, Marshall EJ, Checkley SA. Salivary cortisol measurements during a medically assisted alcohol withdrawal. Addict Biol. 2001;6:247-256.

43. Homann N, Tillonen J, Meurman JH et al. Increased salivary acetaldehyde levels in heavy drinkers and smokers: a microbiological approach to oral cavity cancer. Carcinogenesis. 2000;21:663-668.

44. Khocht A, Schleifer SJ, Janal MN, Keller S. Dental care and oral disease in alcohol dependent persons. J Subst Abuse Treat. 2009;2:214-218.

45. Kobus A, Szajda SD, Kierklo A et al. Oral status in smoking and nonsmoking alcohol abusing patients. Polish J Environ Stud. 2008;17:306-310.

46. Slomiany BL, Liau YH, Zalesna G, Slomiany A. Effect of ethanol on the in vitro sulfation of salivary mucin. Alcohol Clin Exp Res. 1988;12:774-779. 